\title{
Fortalecimiento de la comprensión y producción de textos, uso de la investigación como estrategia pedagógica, con apoyo en tecnologías de información y comunicación ${ }^{1}$
}

Strengtheningtheunderstanding and production of texts,
use of research as a pedagogicalstrategy, withsupport in
information and communicationtechnologies

DOI: http://dx.doi.org/10.17981/cultedusoc.9.3.2018.27

Artículo de investigación. Fecha de recepción: 15/06/2018. Fecha de aceptación: 27/11/2018

Alva Montenegro De Pertuz; Yolanda Cantillo Molina; Celina Pabón Pallares; Maria Gonzales Castañeda; Edgardo Villa Real Martínez; Javier Guerrero Guerrero;

Alfonso Lobato Petuz; Heber López Pérez; Olga Cantillo Bolaño;

Arquímedes Hernández Ariza; Adelso Gómez Hernández; Lucila Polo Sánchez;

Clementina Villamil; Arelys Villa Páez; Emerson Pertuz Samper;

Lila De La Hoz Cera; José Sánchez Paz; Hernando Vega Cantillo;

Rosmari Mozo Carranza; Andres Villafañe Ayala; Yosmar Ropain Caballero;

María De la Cruz; Omar Potes Donado; Rosmira Sánchez y Luxer Serrano Cantillo²

Institución Educativa Departamental Agropecuaria Nuestra Señora de Las Mercedes (Colombia) lividaescorciaruda1971@outlook.com

Para citar este artículo:

Montenegro, A., Cantillo, Y., Pabón, C., Gonzales, M., Villa,E., Guerrero, J., Lobato, A., López, H., Cantillo, O., Hernández, A., Gómez, A., Polo, L., Villamil, C., Villa, A., Pertuz, E., De La Hoz, L., Sánchez, J., Vega, H., Mozo, R., Villafañe, A., Ropain, Y., De la Cruz, M., Potes, O., Sánchez, R. y Serrano, L. (2018). Fortalecimiento de la comprensión y producción de textos, uso de la investigación como estrategia pedagógica, con apoyo en tecnologías de información y comunicación. Cultura. Educación y Sociedad 9(3), 237-246. DOI: http:// dx.doi.org/10.17981/cultedusoc.9.3.2018.27

\section{Resumen}

Este artículo presenta resultados derivados de la investigación acerca del desarrollo de la comprensión lectora y la producción escrita, comocompetencias necesarias en la formación integral de los estudiantes, no sólo del área del lenguaje, sino en todo su quehacer comunicativo e intelectual, en favor de un aprendizaje significativo. El propósito es generar procesos para fortalecer una práctica pedagógica que promuevael desarrollo de aprendizajes basados en la investigación como estrategia pedagógica y la incorporación de los avances de las tecnologías de la información y la comunicación. Se desarrolló la investigación con estudiantes de la Institución Educativa Departamental Agropecuaria Nuestra Señora de las Mercedes, ubicada en Magdalena, Pivijay, Colombia. Se generaron orientaciones para la integración curricular de la investigación y las tecnologías de información y comunicación, para orientar innovaciones necesarias para redefinir las estrategias que adoptan los docentes en su quehacer pedagógico.

Palabras clave: Comprensión lectora, producción textual, Tecnologías de Información y Comunicación, Investigación como Estrategia Pedagógica.

\section{Abstract}

Thisarticlepresentsresultsderivedfromresearchonthedevelopment of readingcomprehension and writtenproduction, as necessarycompetences in the integral formation of students, notonly in thearea of language, but in alltheircommunicative and intellectualwork, in favor of a significantlearningThepurposeistogenerateprocessestostrengthen a pedagogicalpracticethatpromotesthedevelopment of learningbasedonresearch as a pedagogicalstrategy and theincorporation of advances in information and communicationtechnologies.TheresearchwasdevelopedwithstudentsfromtheAgriculturalDepartmentalInstitution of Our Lady of Mercy, located in Magdalena, Pivijay, Colombia. Guidelinesforthe curricular integration of research and information and communicationtechnologiesweregeneratedto guide innovationsnecessaryto redefine thestrategiesadoptedbyteachers in theirpedagogicalwork

Keywords: Reading comprehension, textual production, Information and Communication Technologies, Research as PedagogicalStrategy

1 Este artículo ha sido derivado del Proyecto Fortalecimiento de la Cultura Ciudadana y Democrática en CT+I a través de la IEP apoyada en TIC en el Dpto. del Magdalena.

2 Docentes de la Institución Educativa Departamental Agropecuaria Nuestra Señora de Las Mercedes

- The author; licensee Universidad de la Costa - CUC.

Cultura, Educación y Sociedad vol. 9 no. 3, pp. 237-246. Diciembre, 2018

Barranquilla. ISSN 2389-7724 Online 


\section{Introducción}

La comprensión lectora y la producción de textos son procesos que van de la mano y evidencian el desarrollo de cualidades y competencias que no sólo impactan el área del lenguaje, sino también en el aprendizaje significativo del resto de contenidos trabajados a lo largo de la vida y en la integración del estudiante al contexto educativo. De acuerdo a Valladares (1998), la comprensión de textos se encuentra presente en los distintos escenarios y niveles educativos, representando una actividad fundamental en los procesos de enseñanza-aprendizaje, ya que todo el contenido trabajado en las distintas asignaturas se estudia, analiza y discute a partir de textos escritos.

En este mismo sentido, Gómez (2011), advierte que un bajo nivel en el desarrollo de estos procesos y cualidades,se verá reflejado en un bajo rendimiento académico general, ya que impactan en todas las áreas curriculares de educación primaria: en la comunicación integral, el desarrollo del pensamiento lógico-matemático y en el cultivo de las relaciones: ciencia y ambiente, persona- sociedad y hasta el la formación espiritual y religiosa

En la Institución Educativa Departamental Agropecuaria Nuestra Señora De Las Mercedes, esta fue la preocupación Equipo de Investigación Los Socráticos, quienes notaron el bajo rendimiento que estaban presentando sus estudiantes, y al revisar los resultados de las Pruebas Saber, notaron que presentaban niveles deficientes en el área de lenguaje en las competencias lectoras y muy bajos niveles en el resto de las áreas. De esta manera, el cuerpo docente al relacionar sus prácticas pedagógicas cotidianas con los resultados, y basados en una revisión teórica, relacionaron estas dificultades con carencias en el desarrollo de las competencias de lectura comprensiva y de producción de textos en los estudiantes.
Por otro lado, la discusión inicial en la definición de este estudio, condujo a reconocer características muy tradicionales y estáticas en las prácticas pedagógicas trabajadas en la escuela, de lo cual se tienen evidencias como las siguientes: no se promueve la participación activa y frecuentemente se desarrollan clases de manera magistral. Razón por la cual el equipo consideró necesario buscar alternativas para corregir esta situación, mediante un proceso de innovación curricular que pueda tener pertinencia desde la integración curricular de la Investigación como Estrategia Pedagógica (IEP), y con apoyo de las Tecnologías de la Información y la Comunicación (TIC).

Entendiendo estrategia como un dinamismo que potencializa el crecimiento socioeconómico, que genera desarrollo e inclusión (Pacheco, 2013), y la investigación como estrategia pedagógica debe ser concebida similar al pensamiento estratégico que según Arellano (2004) citado por Mendoza y López (2015), "hace referencia a la forma del pensamiento que se dirige hacia la creatividad, imaginación, y búsqueda de opciones innovadoras y realistas, como potenciación de lo posible enmarcado dentro de un proyecto de construcción intencional de la realidad" (p. 154)

\section{El por qué y para qué de la lectura}

Con el fin de superar estas carencias en los procesos pedagógicos y para promover la comprensión lectora y producción escrita, se asumió como un paso fundamental, en primerainstancia, promover la lectura, ya que es la antesala de la comprensión de textos. Así, leer requiere de una serie de procesos cognitivos que permiten decodificar la información que se encuentra en un texto; sin embargo, va más allá de esto, en tanto el lector no recibe el contenido de manera pasiva, sino que lo analiza y le da sentido en función de sus necesidades, conocimientos 
y experiencias previas, y determina lo que es relevante de ser almacenado (Arteaga, 2001). La lectura que integra ir más allá del texto o la palabra escrita, para detectar intenciones, posturas y contextos donde se encuentra su autor, es el objeto fundamental que mueve la realización de este trabajo.

En este sentido, de acuerdo a Solé (2012), leer de manera comprensiva implica una aprehensión del contenido con criterio, hecha de manera reflexiva, con el fin de seleccionar qué es relevante de ser leído, como aproximarse al texto, y de qué manera hace uso de la información del mismo en la cotidianidad.

Desde la evaluación realizada en las Pruebas Saber, las competencias lectoras incluyen el análisis y reflexión de los contenidos de distintos tipos de texto, teniendo en cuenta su estructura, intencionalidad y aplicación tanto en el marco del contexto educativo como fuera de este (ICFES, 2016).

Tal como se mencionó anteriormente, el desarrollo de competencias en comprensión lectora facilita experiencias de aprendizaje significativo de todos los contenidos y experiencias trabajadas en el aula y fuera de ella, a lo largo de la vida, y puntualmente son muy importantes para contribuir al desarrollo de competencias necesarias para el área del lengua, al tiempo que fortalece la formación integral en cualquier otra del currículum académico. Es la comprensión lectora la que permite realizar un análisis profundo, una lectura crítica de lo aprendido, para que el conocimiento no sea memorístico, sino que sea construido con sentido para el alumno y en interrelación con el contexto (SEP, 2011). Desde estas definiciones que expresan intenciones formativas, se destaca la importancia de la lectura por su impacto en los procesos de aprendizaje significativo, colaborativo e individualmente, para promover el pensamiento y la construcción reflexiva, innovadora y creativa como cualidades fundamentales de un aprendizaje centrado en el alumno, que es la tarea fundamental de la enseñanza.

La lectura y comprensión de un texto puede realizarse en tres niveles (Catalá et al., 2008): uno es el referido a la comprensión literal, en el cual se identifican elementos quese encuentran explícitamente en el texto. Este nivel es más elemental y con más frecuencia trabajado en clase por la mayoría de los docentes. Unsegundo nivel, tiene un carácter inferencial y es un poco más complejo y avanzado que el literal,porque exige el reconocimiento de información implícita en el texto, es decir encontrar la intencionalidad que subyace en el mismo. Finalmente, el nivel crítico, que constituye el nivel más profundo de comprensión, porque requiere un análisis valorativo de lo leído y además exige argumentar su postura o percepción frente a lo leído. En este nivel se observa máxima complejidad, porque expresa la interacción del lector con el texto y su percepción a partir de experiencias personales o bien de argumentos conocidos y fundamentados previamente.

Alrededor de este mismo tema, las destrezas que deben fomentarse en el contexto educativo, para alcanzar un nivel profundo de comprensión. Al respecto, Sánchez (2009), planteauna serie de fases muy importantes: En primer lugar, la necesidad de desarrollar la capacidad de reconocer las palabras leídas con rapidez y precisión. Una segunda fase muy importante, es la de procesar la información con eficiencia y relacionándola con conocimientos previamente alcanzados, de manera coherente; esta es la vía más expedita para alcanzar aprendizaje con sentido y significado. Y una tercera fase, lleva a ejercitar la capacidad de reconocer el tipo de lectura que está realizando, y los juicios que construye el lector, ya que existe una diversidad de tipos de lectura y no es lo mismo cuando se realiza la lectura de una poesía o una novela, a cuando se lee el texto informativo o un blog en internet. 


\section{El proceso de contribuir al desarrollo de la producción escrita}

El proceso que contribuye a desarrollar la habilidad de redactar un texto escrito por parte del que aprende, ha sido trabajado por la UNICEF (2010), organización que plantea:

"la escritura y el acto de escribir adquieren valor epistémico, como instrumento privilegiado del conocimiento, por su potencia para ordenar las ideas y contribuir al esclarecimiento del que escribe, acerca de lo que piensa y sabe sobre un objeto de conocimiento. Cuando el escritor hace el esfuerzo de escribir un texto de esta forma, siente que al terminarlo sabe más que antes, porque jerarquizó y organizó las ideas, porque usó un vocabulario específico y porque pudo explicar un tema", (p. 71).

Existen distintos modelos pedagógicos a partir de los cuales se ha buscado promover, de manera didáctica, la enseñanza de la escritura. Así por ejemplo, Sánchez y Borzone (2010), proponen un modelo integrador, que consiste en tomar en cuenta tanto el desarrollo de las habilidades de nivel inferior, caracterizadas por el procesamiento fonológico: la escritura de las palabras y el trazado, es decir, lo que se necesita para realizar el proceso de transcripción. De igual modo, la fase siguiente, correspondiente a procesos de nivel superior en complejidad, comprende procesos de organización, de jerarquización o esquematización relacionada de las ideas construidas, al tiempo que hace un proceso de revisión o valoración argumentada de todo el proceso vivido o experimentado de manera contextualizada.

De este modo, cumple un papel fundamental las actividades propuestas por los docentes, quienes deben constituirse en guías en la enseñanza de la escritura, estableciendo una relación sinérgica y horizontal con los estudiantes, promoviendo resultados creativos o divergentes y/o resultados convergentes que deben ser evaluados por estudiantes y/o docentes en una relación dialéctica en la que interesan los procesos y los resultados, en la que el profesor y el alumno promueven proponen, guían, evalúan y corrigen.

Lo anterior en el entendido de que la humanidad debe ser formada como sociedad de líderes éticos e integrales que puedan emplear habilidades para consensuar entre miembros de grupos y dirigir acciones por y para un bien común de países, organismos, instituciones entre otros. (Kadi \& Acevedo, 2014)

Un análisis de factores que deben cuidarse al diseñar experiencias formativas en la construcción de textos, lo presentan Reimers y Jacobs (2008), quienes consideran como factores mediadores para lograr el aprendizaje de habilidades para la comprensión y producción escrita, a los siguientes: la motivación, siendo que el interés y la disposición del alumnopuede disminuir por causa de las dificultades en el aprendizaje y la frustración que se genera en el proceso de lograrlo. Por otro lado, hay factores que pueden resultar mediadores determinantes, que provienen del contexto social, como son la participación familiary su contribución a fortalecer la comprensión lectora y la producción escrita. Este es el espacio donde ocurre el clima que promueve la lectura y la autoestima de los estudiantes. En suma, todo el contexto formativo alrededor de los juegos del niño, el uso de su tiempo libre y sobre todo las conexiones emotivas que siembran, con entusiasmo, esos hábitos dentro y fuera del aula.

$\mathrm{Al}$ respecto, en una investigación llevada a cabo por Bevans, et al. (2001), se encontró que involucrar a los padres, fomenta vínculos y compromiso a nivel familiar con el proceso educativo, aumenta el tiempo dedicado a la lectura y mejora la actitud de los estudiantes frente a la misma. Este tipo de estrategiaspromueven prácticas flexibles y voluntarias mucho más atractivas para los estudiantes, en tanto se llevan a cabo por fuera del contexto escolar. 
Desafíos e incorporación de aportes de las Tecnologías de Información y Comunicación

Las políticas educativas que se han venido implementando, buscan promover una educación de calidad a través de la implementación de recursos tecnológicos, que dinamicen los procesos de enseñanza-aprendizaje. En este sentido, "con los recursos que ofrece la tecnología, los procesos de lectura están llamados a sufrir transformaciones, ya que éstas permiten realizar actividades didácticas nuevas y de gran atractivo para los estudiantes, y pueden contribuir eficazmente a mejorar el logro de los objetivos curriculares en general, en especial los relacionados con la lectura y escritura en particular (Clavijo, Maldonado y Sanjuanelo, 2011, p. 27).

La UNESCO (2008), señala que esta era de la tecnología desafía la evolución de una práctica educativa en cuanto a la actualización de su contexto, sus contenidos y los métodos o modelos que se empleen. En este sentido, se requiere un cambio en los procesos de enseñanza-aprendizaje,para generar experiencias donde el estudiante tenga un rol activo e interactivo y la base de la formación pasa a centrarse en el estudiante y en su aprendizaje integral, en un ambiente de horizontalidad y no de jerarquías. Esto trae cambios muy importantes en el currículo y en la gestión de las instituciones desde todo punto de vista, porque deben adaptarse a esta exigencia.

En Colombia, de acuerdo al Plan Nacional Decenal de Educación 2016-2026 (MEN, 2017), se han planteado lineamientos educativos en los que las instituciones educativas tienen un nuevo desafío y es el replanteamiento de las prácticas pedagógicas tradicionales, como un camino de posibilidades y alternativas para mejorar las prácticas educativas aprovechando los aportes y escenarios interactivos que abren las TIC.
Para Marqués (2013), las TIC plantean muchos desafíos que hay que tomar en cuenta en la implementación de la práctica educativa renovada, así como presenta también beneficiosen los procesos que se desarrollan. Así, dentro de las funciones de las TIC en la educación se encuentra que se presentan nuevos canales de comunicación entre los docentes y los estudiantes, requiere del ejercicio de la vinculación a través de un medio fundamentalmente escrito, pero también cuenta con la construcción y el aprovechamiento de la imagen visual y auditivo. Esta es la era del aprovechamiento del medio digital y el formato web, como un instrumento para promover diversidad de competencias comunicacionales en el alumno al procesar la información. El utilizarlos como un medio didáctico para el logro de conductas previstas en el diseño curricular, sirve como guía de un aprendizaje integral y desde los diferentes estilos, una posibilidad para multiplicar y enriquecer el rol docente y como generador de nuevos escenarios formativos.

\section{La Investigación en el aprendizaje, desde los aportes y desafíos de las TIC}

El uso de la diversidad de herramientas tecnológicas, de la mano con la IEP como estrategia metodológica en el contexto educativo, le abren camino de gran alcance y le permite sembrar procesos colaborativos entre los distintos actores, docentes y estudiantes, que se pueden dar en la virtualidad y que potencia los procesos investigativos y de aprendizaje significativo (Mejía y Manjarréz, 2011). Muchos de esos procesos toman fuerza cuando se considera la diversidad de escenarios y competencias que entran en juego en el proceso de intercambio multicultural, la toma de decisiones que hace el alumno, tanto en individual como en lo colectivo. 
Indudablemente que el cumplimiento de estas promesas tecnológicas en los procesos formativos, a través de la indagación en el aprendizaje, está mediada por las posibilidades de acceso a tal diversidad de recursos y/o por las consecuencias que tal diversidad de información se constituya en un distractor para el alumno, en lugar de guiarlo de la mano. Este es un desafío muy importante al cual los docentes deben saber construir espacios y procesos pedagógicos que aprovechen sus ventajas y minimicen sus riesgos.

\section{Metodología}

El artículo que se presenta es producto del estudio del fortalecimiento de la comprensión lectora y la producción de textos, desde el uso de la IEP y apoyada en las TIC; el cual se desarrolló en la Institución Educativa Departamental Agropecuaria Nuestra Señora De Las Mercedes; con los siguientes rasgos metodológicos:

El diseño: El estudio tuvo una metodología mixta, se estudiaron las cifras resultantes de la aplicación de las Priebas Saber y también se aplicaron experiencias con mayor énfasis en lo cualitativo, la investigación - acción. Se realizó un seguimiento a experiencias diseñadas y aplicadasen la población de estudio, para provocar el desarrollo de la comprensión lectora y la construcción de textos impresos, en los alumnos.

Se realizó una revisión de los resultados de las Pruebas Saber 2016, para $3^{\circ}, 5^{\circ}$ y $9^{\circ}$ en el área del lenguaje. Así mismo, se llevó a cabo un registro y valoración de las competencias lecto-escritoras de la población de estudio, en las prácticas educativas cotidianas, para verificar los resultados de las pruebas:

Procedimiento: Se construyó, a partir de los datos de las pruebas y el seguimiento en el aula, una propuesta pedagógica, tal como se describe: Se promovió la creación de semilleros o grupos de redes temáticas, en distintas áreas del conocimiento, propiciando entre los estudiantes preguntas de investigación en torno a intereses comunes. El proceso vivido en la experiencia se registraba y el resultado de estos encuentros de lectura y debate se analizó como evidencias del proceso de relatorías en cada encuentro.

Así mismo, se aplicó la estrategia "El libro Viajero" mediante la cual los relatos e historias escritas por los estudiantes, resultado de sus encuentros en semilleros, eran depositados en una maleta, la cual era trasladada entre las distintas sedes de la escuela, permitiendo la socialización de los elementos producidos entre los estudiantes, así como la introducción de nuevos contenidos en las clases.

Otra estrategia implementada fue "Mi maleta lectora", en la cual se colocaron distintos tipos de cuentos y lecturas para rotar por las familias durante dos días, de manera que pudiesen leerlos en casa y hacer un análisis de estos con sus padres. Esto con el fin de motivar tanto a los niños y niñas de la clase, como a las propias familias a compartir momentos de lectura, comunicación y afecto, además de promover el protagonismo alos estudiantes dentro de la actividad. Esta experiencia promovió la expresión, en un contexto más cercano, sus opiniones, sentimientos y emociones.

Finalmente, se realizaron espacios de socialización de las actividades desarrolladas y mediante la participación activa los estudiantes, quienes dieron sus recomendaciones en relación a la implementación de las TIC, en el proceso para darle mayor dinamismo y contribuir al mejoramiento de la comprensión lectora y la producción escrita.

\section{Resultados}

Del análisis de los resultados en las Pruebas Saber 2016, se destacan los siguientes gráficos: 


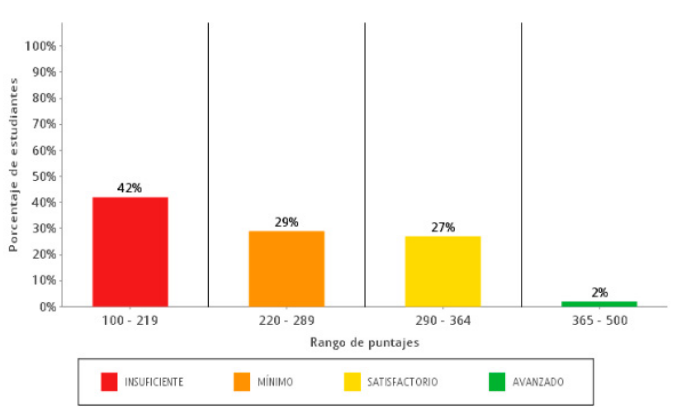

Figura 1. Resultados $3^{\circ}$ Área de Lenguaje

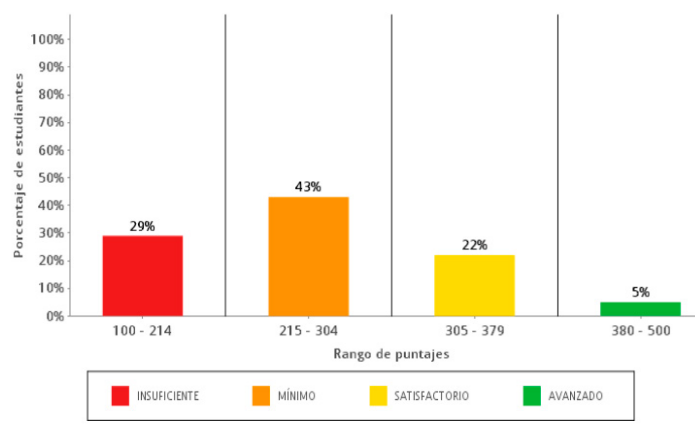

Figura 2. Resultados $5^{\circ}$ Área de Lenguaje

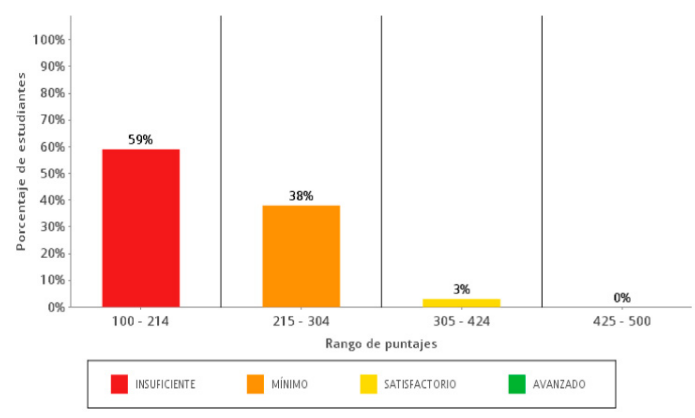

Figura 3. Resultados $9^{\circ}$ Área de Lenguaje

En los gráficos se observa que tanto para los años $3^{\circ}$ (Figura 1 ), $5^{\circ}$ (Figura 2) y $9^{\circ}$ (Figura 3 ), en el área de lenguaje, se evidencian ponderaciones insuficientes para el promedio de alumnos, siendo un poco más favorable los resultados del grado $5^{\circ}$ en el que este resultado de insuficiencia no supera el $30 \%$, aunque ello no significa buen nivel de calidad en el desempeño de esta área.
En cuanto a la aplicación de las estrategias, se realizaron grupos de trabajo en cada grado de la población de estudio, en los cuales se manejaron lecturas dirigidas sobre preguntas problemas surgidas del interés común del grupo. Es importante resaltar que el propósito de la lectura que responde a un tema de interés investigativo, como proyecto de aula, se prevé la integración curricular de la IEP como estrategia funcional a la solución de la problemática, en torno a la comprensión lectora y producción de texto; los cuales fueron generados desde la realización de relatos construidos por los estudiantes en torno al tema y socializados para generar retrospectiva y aportes, que se pudieran propiciar entre los estudiantes participes,para alcanzar el desarrollo de habilidades en las competencias comunicativas que son nuestro eje de interés.

La estrategia denominada "El libro viajero" permitió dinamizar con interacciones diversas, los espacios de aprendizaje, al recibir a manera de correspondencias, las producciones de estudiantes de otras sedes. El proceso de retroalimentación de los mismos fue muy activo y logró que se sintieran empoderados de su proceso de aprendizaje. Asimismo, la estrategia " $\mathrm{Mi}$ maleta lectora" generó una excelente acogida entre los estudiantes participes, durante esta actividad la maleta lectora. La participación activa de los padres se hizo evidente mediante la evidencia del documento escrito adjunto que debían incluir de regreso como resultado de su proceso en el hogar.

\section{Conclusiones}

El desarrollo de proyectos de investigación como estrategia pedagógica, generado en los procesos de aprendizaje, y apoyado con las TIC, resulta un mecanismo que se integra a la gestión de experiencias que se insertan en el currículo, para promover el desarrollo de competencias comunicacionales. 
Constituye una alternativa de redefinición e innovación de las estrategias que adoptan los docentes para fortalecer su quehacer pedagógico, desde la práctica.

Los resultados de la experiencia del trabajo realizado permitieron un contraste entre las competencias que posee la población de estudiantes, respecto al área de lenguaje, así como se manifiesta en su motivación para trabajar los contenidos planteados en cada área, lo cual pudo ser constatado por los docentes en el desarrollo de las clases.

Para el diseño de estrategias con fines de fortalecer la compresión lectora, es indispensable, que parte de estos procesos sean trabajados desde la niñez; por esoel MEN (2017), plantea que son conscientes de que el proceso de aprendizaje, especialmente en los primeros años, deja en el niño una huella que perdura toda su vida. Por lo tanto, este proceso debe ser una experiencia agradable, llena de sentido y significado, donde no se generen angustias frente a las equivocaciones, sino que éstas sirvan para fortalecer el aprendizaje.

Esto da claridad y entrada a que la utilización delos aportes de las TIC, constituyen un medio por el cual se pueden diseñar diversidad de estrategias con fines educativos, debe ser de manera dinámica y acogedora, pertinente al aprendizaje de los niños, para potenciar en ellos buenos hábitos de lectura y así también desarrollar un pensamiento crítico, para las competencias que hoy se establecen como lineamientos educativos generales, según los estándares colombianos.

Por otro lado, es importante puntualizar que "se favorece el proceso de aprendizaje significativo,cuando las ideas expresadas simbólicamente son relacionadas de modo no arbitrario, sino sustancial (no al pie de la letra), con lo que el estudiante ya sabe, en cuanto a sus conocimientos y experiencias previas. Por consiguiente, al hacer referencia al proceso de aprendizaje de la lectura, se estima que este depende de qué tanto el individuo domina de manera previa el lenguaje hablado y que éste a su vez sirva como soporte para la percepción de significados del lenguaje escrito" (Franco, 2011, p. 76).

Por último, se considera que la aplicación de las distintas estrategias utilizadas como la del libro viajero, la maleta lectora y el proyecto de investigación escolar desarrollado a través de los semilleros, corresponden a mecanismos de integración curricular de la IEP, siendo muy importante resaltar que se fortalecieron las habilidades en los estudiantes respecto a la interpretación y producción de textos, en las distintas áreas de trabajo en la institución y, para efectos de una mayor trascendencia del proyecto. Se requiere una continuidad en la aplicación y mejoramiento de las estrategias, con los respectivos indicadores de impacto a partir de los elementos de valoración que permitan cuantificarlo. Sólo quedaría entonces, identificar otros mecanismos para ayudar a construir una institucionalidad escolar que garantice un desarrollo oportuno de la comprensión y producción de textos. (Amézquita Zárate, 2014)

\section{Referencias}

Amézquita, P. (2014). Minería y petróleo en Colombia: maldición interna de los recursos. Económicas CUC, 35(1), 4559.

Arteaga, I. (2001). Comprensión Lectora. Lima, Perú.

Bevans, B., Furnish, B., Ramsey, A., \& Talsma, S. (2001), Effective strategies for homeschool partnerships in reading. Chicago: Saint Xavier University and SkyLight Professional Development Field-Based Masters Program. 
Catalá, G., Catalá, M.; Molina, E. y Monclús, R. (2008). Evaluación de la Comprensión Lectora. Pruebas ACL $\left(1^{\circ}-6^{o}\right.$ de primaria). Barcelona: GRAÓ.

Clavijo, J., Maldonado, A. y Sanjuanelo, M. (2011) Potenciar la comprensión lectora desde la tecnología de la información. Revista Escenarios, 9(2). 26 -36. Consultado en http://hdl.handle. net/11619/1619

Gómez, J. (2011). Comprensión lectora y rendimiento escolar: una ruta para mejorar la comunicación. COMUNI@ CCIÓN: Revista de Investigación en Comunicación y Desarrollo, 2(2), p. 27-36.

Franco, M. (2011). Intervención psico-educativa para la promoción de la comprensión lectora. Cultura, Educación y Sociedad, 2(1). 75-84.

ICFES (2016). Guía de Interpretación y Uso de Resultados de las pruebas Saber $3^{\circ}, 5^{\circ}$ y $9^{\circ}$. Bogotá D.C.: Ministerio de Educación Nacional.

Kadi , O. y Acevedo, Á. E. (2014). Liderazgo ético frente a la diversidad cultural dentro de las organizaciones con régimen disciplinario. Económicas CUC, 35(2), 75-88.

Marqués, P. (2013). Impacto de las TIC en la educación: funciones y limitaciones. 3 c TIC: cuadernos de desarrollo aplicados a las TIC, 2(1), 2.

Mejía, M. y Manjarrés, M. (2011). La investigación como estrategia pedagógica. Una apuesta por construir pedagogías críticas en el Siglo XXI. Praxix\& Saber, 2(4), 127-177

MEN (2017). Ruta de apropiación de TIC en el Desarrollo Profesional Docente. Bogotá D.C.: Ministerio de Educación Nacional Colombia. Programa Nacional de uso de Medios y Nuevas Tecnologías. Recuperado de http://www. plandecenal.edu.co/cms/media/herramientas/PNDE\%20FINAL_ISBN\%20 web.pdf.
Mendoza, D. y López, D. 2015). Pensamiento estratégico: centro neurálgico de la planificación estratégica que transforma la visión en acción. Económicas CUC, 36(1), 153-179.

Pacheco, C. (2013). Estrategias empresariales más utilizadas por las pymes en Sincelejo. Económicas CUC, 34(1), 183-202.

Reimers, F. y Jacobs, J. (2008), La lectura en la sociedad de la información. Leer (comprender y aprender) y escribir para comunicarse. Desafíos y oportunidades para los sistemas educativos. XXIII Semana Monográfica de la Educación. Documento Básico: OEI. Consultado en https://www.google.com.co/search? $q=($ Reimers $+y+J a$ cobs\%2C+2008).\&rlz=1C1CHBF_es CO801CO801\&oq $=($ Reimers $+y+J a c o$ bs\%2C+2008).\&aqs =chrome..69i57.4 $543 \mathrm{j} 0 \mathrm{j} 8 \&$ sourceid $=$ chrome $\& \mathrm{ie}=\mathrm{U}$ TF-8

Sánchez, E. (2009). Ayudar a comprender y enseñar a comprender. Necesidades de los alumnos y necesidades de los profesores. En, Ministerio de Educación, Congreso Leer.es, Madrid.

Sánchez, V. y Borzone, A. (2010). Enseñar a escribir textos: desde los modelos de escritura práctica en el aula. Lectura y vida, 31(1).

SEP. (2011). Manual de procedimiento para el fomento y la valoración de la competencia lectora en el Aula. México. D.F.: Secretaría de Educación Pública

Solé, I. (2012). Competencia lectora y aprendizaje. Revista Iberoamericana de Educación (OEI), (59), p. 43-61.

UNESCO. (2008). Declaración mundial sobre educación para todos. Satisfacción de las Necesidades Básicas de Aprendizaje. Jomtien, Tailandia. 
UNICEF. (2010). Una Escuela Secundaria Obligatoria para todos - Cuaderno para profesores. Buenos Aires: Educación para todos
Valladares, O. (1998). Comunicación Integral. Bases Técnicas y Desarrollo de Competencias Comunicativas. [Tesis bachiller]. Universidad Nacional de Huancavelica, Huancavelica. 